\title{
Life-cycle assessment framework for indoor emissions of synthetic nanoparticles
}

\author{
Tobias Walser $\mathbb{D}^{-}$David Meyer • \\ Wouter Fransman · Harrie Buist • \\ Eelco Kuijpers • Derk Brouwer
}

Received: 13 October 2014/ Accepted: 26 May 2015/Published online: 3 June 2015

(C) Springer Science+Business Media Dordrecht 2015

\begin{abstract}
Life-Cycle Assessment (LCA) is a wellestablished method to evaluate impacts of chemicals on the environment and human health along the lifespan of products. However, the increasingly produced and applied nanomaterials (defined as one dimension $<100 \mathrm{~nm}$ ) show particular characteristics which are different from conventional chemicals or larger particles. As a consequence, LCA does not provide sufficient guidance on how to deal with synthetic nanomaterials, neither in the exposure, nor in the effect assessment. This is particularly true for the workplace, where significant exposure can be expected via the lung, the route of major concern. Therefore, we developed a concise method which allows the inclusion of indoor nanoparticle
\end{abstract}

Electronic supplementary material The online version of this article (doi:10.1007/s11051-015-3053-y) contains supplementary material, which is available to authorized users.

T. Walser $(\square)$

Institute for Environmental Engineering, ETH Zurich, 8093 Zurich, Switzerland

e-mail: tobias.walser@bag.admin.ch

T. Walser

Federal Office of Public Health, 3003 Berne, Switzerland

D. Meyer

National Risk Management Research Laboratory,

U.S. Environmental Protection Agency, Cincinnati, OH 45268, USA

W. Fransman - H. Buist · E. Kuijpers · D. Brouwer TNO, P.O. Box 360, 3700 AJ Zeist, The Netherlands exposure into LCA. New nanospecific properties are included along the LCA stages with a particular focus on the workplace environment. We built upon existing LCA methods and nanoparticle fate and exposure studies. The impact assessment requires new approaches for nanoparticles, such as guidance on relevant endpoints, nanospecific properties that are relevant for the toxicity, and guidance on the chemical identity of nanomaterials, i.e., categorization and distinction of different forms of nanomaterials. We present a framework which goes beyond traditional approaches of LCA and includes nanospecific fate parameters in the indoor exposure assessment as well as guidance on the development of effect and characterization factors for inhaled nanoparticles. Specifically, the indoor one-box model is amended with new particle-specific parameters developed in the exposure literature. A concentration conversion and parameter estimation tool are presented. Finally, the modification of the traditional intake fraction to capture size-specific deposition and retention rate are discussed along with a strategy for a more robust effect assessment. The paper is a further step toward a fair comparison between conventional and nano-enabled products by integrating occupational exposure to synthetic nanomaterials into LCA.

Keywords Life cycle assessment (LCA) - Indoor exposure $\cdot$ Synthetic nanoparticle $\cdot$ Nanoparticle toxicity $\cdot$ Occupational exposure $\cdot$ Engineered nanoparticle $\cdot$ Environmental and health effects 


\section{Introduction}

Nanoparticles (NPs) and nanofibers (NFs) are omnipresent in the natural and man-made worlds (Godish and Spengler 1996) and usually defined having at least one dimension $<100 \mathrm{~nm}$ (SCENIHR 2010). Relatively new is the human ability to design synthetic NPs that allow unique functionalization of products. The exponentially growing NP production industry develops NPs which are finding their ways into many nanoenabled intermediate and consumer products. At the same time, there is research to understand the fate and impacts of NPs throughout their life cycle: before, during and after their intended function. Unique properties of NPs, such as high particle number or surface area per equivalent mass or size, increase their reactivity and hence potential toxicity in comparison to the bulk counterparts. Small particles are also taken up by non-phagocytotic cells which can cause adverse cell effects. Therefore, we suggest using the term "nanoscale" for particles up to $500 \mathrm{~nm}$ because endocytosis has been demonstrated for particles up to this size (Rejman et al. 2004). Facilities that produce or handle nanopowders or products that contain nanomaterials are of primary concern for elevated individual human exposure, while NP emissions from the use of nano-enabled products usually lead to lower exposures but potentially affect a higher number of people. Although dermal contact or ingestion of secondary NPs that have settled on surfaces or food and liquids are an area of concern, inhalation is the primary intake route in order to ensure worker safety. The lung deposition pattern and the resulting dose of the inhaled NPs in the respiratory tract and ultimately in bodily organs are influencing the magnitude of health effects. Consequently, occupational exposure limits for NPs are emerging. The US National Institute for Occupational Safety and Health (NIOSH) recently established Recommended Exposure Limits (REL) for nanoscale titanium dioxide $\left(\right.$ nanoTiO $\left.{ }_{2}\right) \quad\left(0.3 \mathrm{mg} / \mathrm{m}^{3}\right)$ and carbon nanotubes (CNTs, $1 \mu \mathrm{g} / \mathrm{m}^{3}$ ) (National Institute for Occupational Safety and Health NIOSH 2013). Complying with such limits minimizes the probability of long-term health effects. However, the lack of harmonization and standardization in the toxicity literature does not allow straight forward risk assessment for NPs. Thus, alternative approaches are required which can support a safe production, use, and disposal of NPs/NFs. More ideally even, the potential human health impacts of nano-enabled products should be put into a larger context to support sustainable development of nanoenabled applications and products. This would include quantifying the benefits and disadvantages of nanoenabled products across multiple impact categories like human toxicity, ecotoxicity, global warming potential, and others. Such an approach is possible through the use of Life-Cycle Assessment (LCA), an environmental assessment tool that can connect human health issues with larger environmental sustainability issues such as climate change, resource conservation, and ecosystems protection. However, LCA does not provide an absolute understanding of health risks because of its comparative character and many simplifying assumptions (e.g., linear extrapolations over time and across exposed populations for dose and effects). In contrast to Risk Assessment (RA), where specific spatial and temporal information of all system elements are required, an LCA can deal with a reduced description of the physico-chemical properties and potential exposure and toxicity pathways of NPs. Even though simplified human health models are used, LCA practitioners still require data on NP emissions, fate, and adverse effects along the life cycle of a nanoenabled product to evaluate the human health impacts.

In this paper, we introduce a framework that accounts for Life-Cycle Inventory (LCI), exposure, and human health impacts caused by inhalation of nanoparticles in indoor environments. It can be used for the assessment of services or products that contain synthetic NPs. The framework uses features of the USEtox model (Rosenbaum et al. 2008) with the recently added indoor compartment and extends it to synthetic NPs. As with other life-cycle impact models, the impact score is the product of an LCI emission flow (EmF, added by the user) and a Characterization Factor (CF) (Rosenbaum et al. 2007). A CF can be further decomposed into an intake fraction (IF) that accounts for where and how a chemical exposure occurs and an Effects Factor (EF) that describes the biological outcome of the exposure. A primary drawback to the USEtox model is that it is only adequate for good mixing conditions, and not for the single-source near-field exposures typically encountered for a variety of chemical and consumer product exposure scenarios (Schneider et al. 2011). In addition, the USEtox model was developed for mass-based 
emissions and not for other units such as surface area or number concentration that might be more appropriate for NPs. For indoor exposure to NPs, the underlying model in USEtox would predict an ambient indoor NP concentration per unit of emitted NP and subsequently translate this concentration to an inhaled intake fraction using an uptake factor. Traditional Intake fractions for PM10 and PM2.5 do not include the size- and shape-dependent deposition and retention capacity of the lung but assume total uptake of the inhaled particles (Humbert et al. 2011). Given the unique properties of NPs, NP-specific uptake factors might be necessary for the various types of NPs. The predicted human exposure to NPs per unit of emitted $\mathrm{NP}$ would then be multiplied with an appropriate EF to obtain a CF for NP emissions. While one can find CFs for microparticles (PM10, PM2.5) in the literature (Jolliet et al. 2003; van Zelm et al. 2008), neither NPs in general nor their synthetic forms are covered in the current LCA framework. The nanospecific CF, once available, would quantify the total impacts on human health per unit of NP emitted. CFs are substancespecific, differ between environmental compartments (Guinée et al. 2001), and generally have a lower level of sophistication than risk quotients with a higher temporal and spatial resolution. The unit of $\mathrm{CF}$ for human health typically is 'cases per $\mathrm{kg}$ pollutant emitted,' preferably based on epidemiologic data of the affected population. Further information on calculation of CFs is found in Rosenbaum (Huijbregts et al. 2005; Rosenbaum et al. 2008) and Huijbregts(Huijbregts et al. 2005). Given the relative newness of synthetic NPs, epidemiologic data are not yet available and in vivo (animal) toxicity data are still scarce. There is intense research on high-throughput assay methods (in vitro) because they would reduce the immense costs for in vivo studies and would facilitate a comparative and robust testing of relevant endpoints for a myriad of NPs. However, the more abundant in vitro data cannot be used today to calculate EFs because reliable conversion methods and factors are lacking.

For the framework developed here, we aim for a pragmatic, yet scientifically justified method that primarily includes worker exposure to nanoparticles in an indoor scenario with potential application to consumer exposure. The parameterization of the model is done systematically by using a hierarchical structure of preferred real measurement data (indoor
NP measurements, chronic toxicity tests, etc.) supplemented with estimated data gaps (worker exposure models, comparable scenarios, conversions of different toxicity studies, etc.). Users will have the flexibility to account for exposure scenarios based on available information by adapting the model parameters as needed. A discussion of uncertainties and limitations will support critical interpretation of NP CFs, while highlighting ongoing research discussions regarding NP exposure and toxicity that are relevant for LCA developers and users.

\section{LCA framework for indoor exposure to nanoparticles}

\section{Framework overview}

Four major contributions are being made to conventional human health methods for LCA in the proposed seven-step-framework (Fig. 1) in order to integrate current knowledge of exposure science and nanotoxicology. First, it is proposed that a set of technology and activity-specific interim EmFs is developed which would provide LCA practitioners generic estimates of NP emissions when developing a life-cycle inventory (LCI, step 1-4). If, for example, the production of a nano-enabled product requires synthetic NPs produced by flame spray pyrolysis (FSP) and actual air monitoring data are unavailable from the FSP facility, and the EmF database would provide a quantified nanomaterial emission in the FSP LCI by suggesting a mass generation rate for a typical unit operation scenario. Using this approach, activity-level knowledge is provided of the impacts of NP emissions, covering not only production of NPs, but also handling activities in the manufacturing, use, and disposal phases. Second, a module has been developed to convert concentration metrics when primary data are available for any step in the framework because of the variability of formats used to report results for exposure and toxicity studies. Third, the indoor model in USEtox, developed primarily for organic compounds, is being adapted to account for NP-specific transport, exposure, uptake, and effects (steps 3-5). A notable change is that uptake rates of NPs by inhalation will rely on fractional uptakes of less than $100 \%$. To account for the population-based nature of LCA human health impacts, estimates for the number 
Nanoparticle emissions from the production of $1 \mathrm{~kg}$ nanoparticles

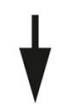

Exposure Scenarios: State of the art; Average production standard; Emission accidents; Handling/Packaging, etc.

\section{Required Parameters}

Physical properties of NP:

Mode of size distribution (aero/hydrodynamic diameter); density; shape; concentration of synthetic and by-product NP

Indoor volume, ventilation rate, agglomeration and sedimentation rate, emission rate

Worker number, inhalation rate, exposure time

convert to total inhaled NP over the life time of the exposed persons

Inhalable fraction which is deposited and retained in the lung (phys-chem specific) convert to total absorbed NP over the life time of the exposed persons

Physic-chemical properties of NP and their mode of toxic action

Concentration-response from nanotoxicity studies (preferably long-term inhalation),

local and systemic effects from effective dose, preferably organ-specific, flag carcinogenicity

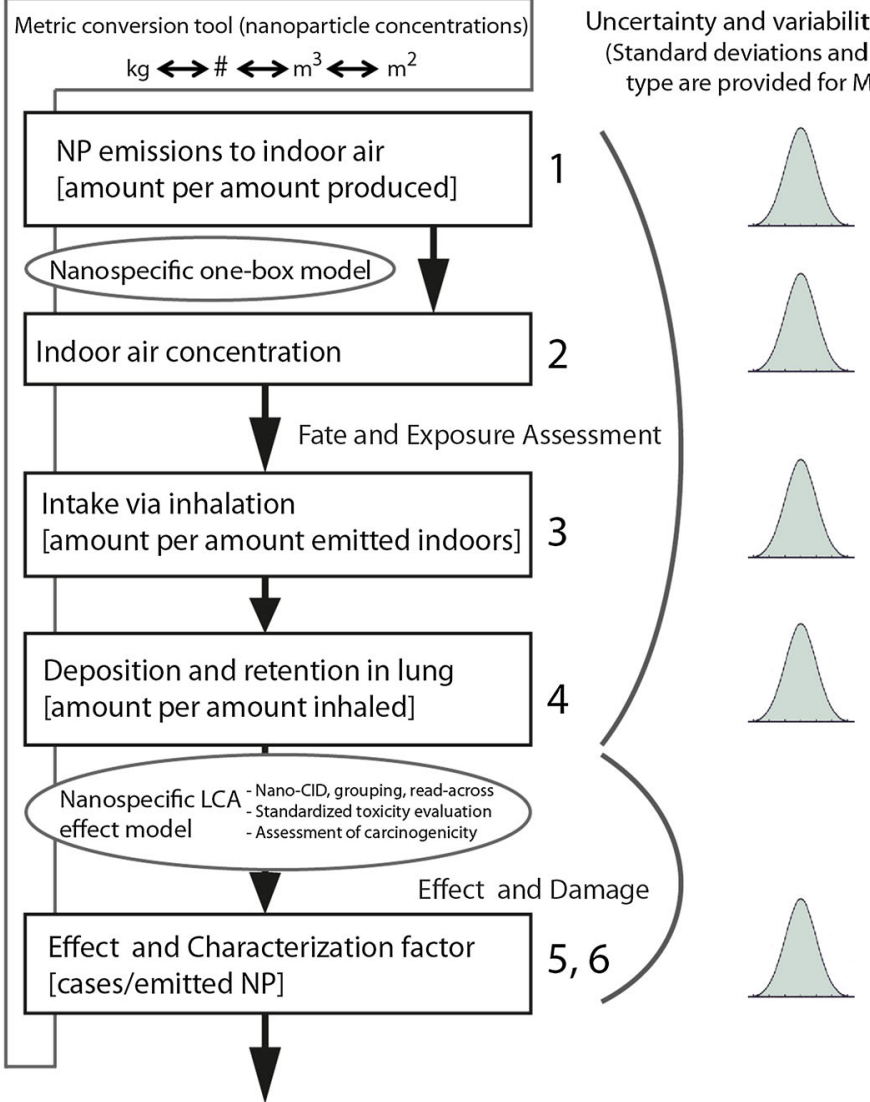

\section{Human toxicity from indoor emissions from the production of $1 \mathrm{~kg}$ nanoparticles}

Fig. 1 LCA framework for the human toxicity assessment of nanoparticle (NP) emissions indoors. Numbers indicate the steps which are further explained in the respective chapters. "Required parameters" describe the necessary information which is needed to run a full LCIA of synthetic nanoparticles

of exposed workers, exposure time, and human factors are part of the framework. Finally, EFs for synthetic NPs are challenging to quantify when compared to similar efforts for conventional chemicals because of the much greater uncertainty associated with nanotoxicology data and the myriad of different NPs. Therefore, guidance being included in this framework will assist with the calculation and interpretation of nanospecific CFs with quantified uncertainty that can be included in LCA uncertainty analysis based on Monte Carlo simulation (steps 5-7). While the (indoors). Ideally, default (but adaptable) values will be provided for the parameters. The framework allows a.o. a free scaling of the amount of produced NP as well as a conversion of metrics at any stage. $M C$ Monte Carlo, CID Chemical IDentity

framework is presented with only limited quantitative information, case studies in a forthcoming paper will illustrate the method presented here.

STEP 1: Nanoparticle emission factors in the workplaces

Worker exposure studies have covered a variety of processes and handling activities (Fig. 2) across various stages of the life cycle of synthetic NPs and CNTs (Fig. 2, Table A5a-m). Usually, the reported 

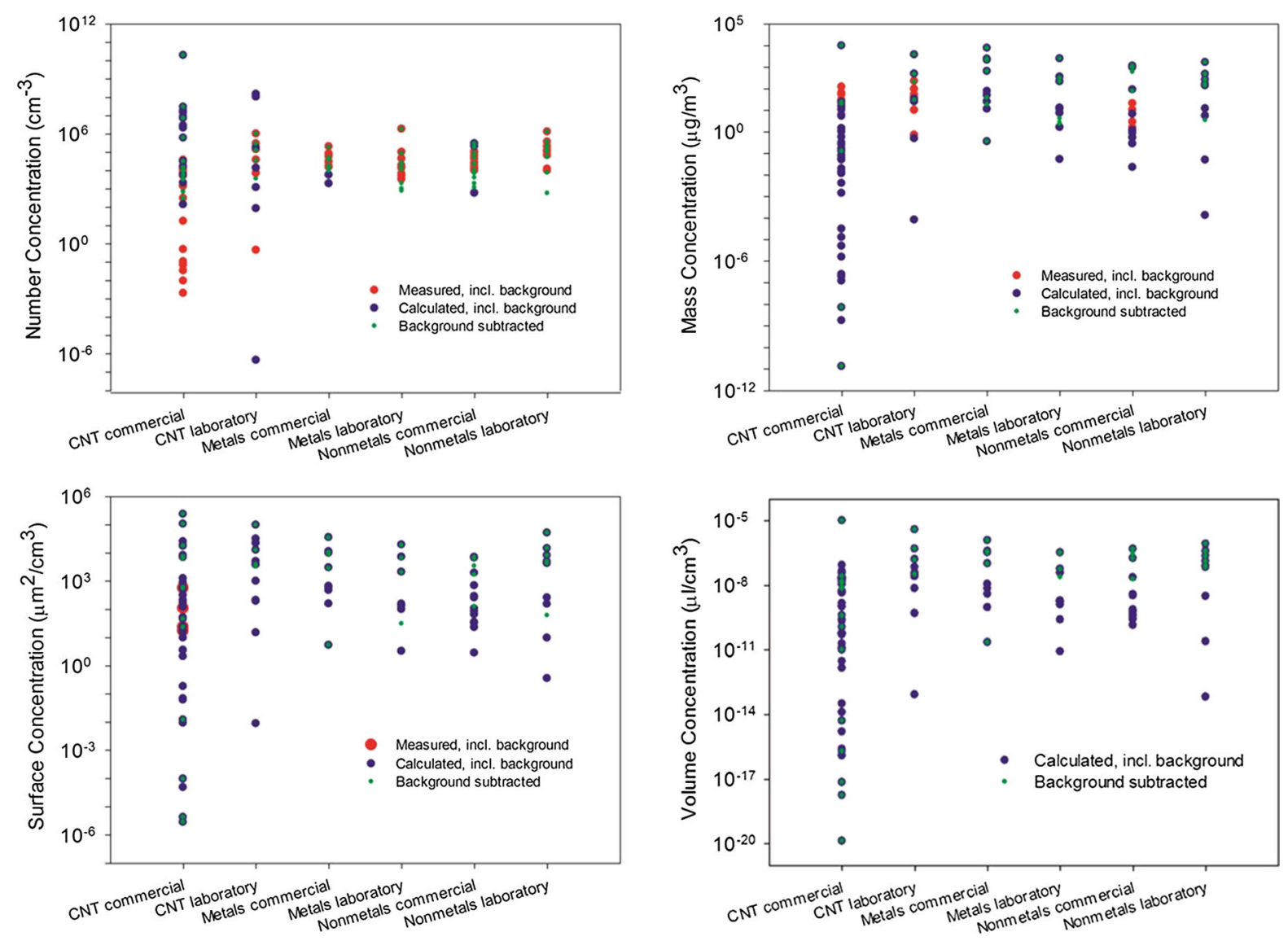

Fig. 2 Indoor NP (nanoparticle) concentrations during production or handling of nanomaterials (unit conversions with the converter tool). The classes distinguish between large industry facilities and laboratory conditions, as well as different

studies focus primarily on investigating the personal exposure level in the breathing zone of a worker, and EmFs are reported by only a handful of studies for limited activities, which does not allow for calculation of generalized EmFs for the different production technologies and activities. Consequently, the ability to include NP emissions in LCI is extremely limited at this time. Therefore, generic EmFs are needed for the different NP production and handling activities. Ideally, such factors will be based on harmonized or, if possible, standardized occupational exposure studies that report synthetic NP emissions (either in a real workplace or by using a simulated controlled environment) for specific technologies and activities. In the absence of these data, EmFs may be estimated from published studies on general NP concentrations in workplaces (Fig. 2), provided the airborne NP data can be related to a corresponding production rate, i.e.,

materials: CNT (carbon nanotubes), metals, and nonmetals. Estimated concentrations are based on measurement results (red) and calculated with the unit converter. References can be found in Table A5a-m. (Color figure online)

the quantity of NPs emitted indoors per quantity of NPs produced. If even these data are lacking, EmFs may be estimated by using quantitative exposure/ emission models. However, the currently available exposure assessment models for NPs are qualitative and give a relative ranking of exposure scenarios rather than a quantitative estimate of emission or exposure. There are some quantitative exposure assessment models available for estimating exposure to chemicals agents, but these are not NP-specific and have not been calibrated/validated with nanoexposure measurement data. Calculation of emission rates based on indoor concentrations has been demonstrated by Walser et al. (2012). Ideally, for the LCA practitioner, the generic NP indoor concentrations will be parameterized for room ventilation rates and room volumes for known manufacturing activities to allow them to be adjusted to better fit a given life-cycle model. 
Unfortunately, the use of results from indoor concentration measurements in back calculations to obtain emission rates can contain a number of critical issues, and at this point in time, we cannot yet present generic emission factors. However, we provide here tools and recommendations on critical issues when interpreting exposure studies and when calculating specific EmFs.

A plethora of ambient NP monitoring methods exist which cover a range of particle sizes and concentrations because no internationally harmonized measurement approach has currently been established. A main obstacle is the difficulty to distinguish between the concentration of background NP and synthetic NP which is difficult to do in real time. In addition, studies on the fate of NPs in workplaces report their results in either mass, surface area, or number concentration and vary in accuracy, precision, and measurement period (Table A5a-m). A conversion into the different units leads to variability of orders-of-magnitude, based on the given limited characterization of the nanomaterials and imprecise distinction between different nanomaterials (Fig. 2). The morphology (shape, density, characteristic length, etc.) and size distribution of the NPs must be known prior to any unit conversion (Figure A1, Table A2). Therefore, a stringent quality check of the measurement data is necessary in order to combine physico-chemical property data for NPs with measurement data. Particular caution must be exercised because a slight decrease in the characteristic length (e.g., NP diameter) beyond a cut-off value can cause an exponential divergence of the number, mass, surface, and volume concentrations (Figure A1). Illustrating it with an example, different measurement techniques, uncertainties in the concentration measurements or in the characterization of material properties (density, diameter, etc.) for NP diameters less than $50 \mathrm{~nm}$ will increase the uncertainty of the conversion results tremendously. When converting from number concentration to mass concentration, most devices apply unit density, which is only an approximation. For example, the specific density of an aerosol is only equal to the density of a solid spherical particle when the fractal dimension equals three. While the conversion tool has been introduced to assist the calculation of EmFs and ultimately indoor concentrations, it is recommended to apply the tool throughout the framework to maintain consistency in exposure and toxicity modeling, acknowledging, that accuracy might not be the best.
STEP 2: Modeling the indoor fate of nanoparticles to derive indoor concentrations

Point sources of NPs in workplaces with poor ventilation can lead to discrepancies between personal exposure to NPs and area concentrations in workplaces (Birch et al. 2011). Therefore, indoor exposure measurements (Fig. 2) have to be analyzed with care when using indoor concentrations for human exposure modeling. NP concentrations can be modeled from emission rates using basic transport models if certain indoor parameters are known. As was discussed in step 1, NP emission rates are dependent on multiple factors such as production technology, production rate, and control measures, making it difficult to report generalized values. Moreover, the usually assumed steady-state concentrations in LCA deviate from the fact that NP emissions are often finite events, and any change in the emission rate significantly influences the NP concentrations. In such a case, time-dependent concentrations and correction for personal protection measures lead to more realistic estimates of cumulative long-term exposure (Golsteijn et al. 2014). Hence, if detailed information is available, an exposure scenario-specific approach provides more accurate results than the traditional LCA approach. Complex (two-box) models exist for describing dispersion of air through the indoor compartment (Meesters et al. 2014; Cherrie et al. 2011). However, if the emission rate does not change significantly over time and concentration gradients in the indoor environment are flat, one-box models provide a good first approximation of NP indoor concentrations and are indicative enough for the purposes of LCA fate models (Hellweg et al. 2009; Walser et al. 2012). Furthermore, the direct compatibility with already used one-box models in LCA and the simplicity of this one-box model in comparison with more complex models (without making too much compromise regarding accuracy in view of the overall uncertainties of LCA) are reason for selection and nanospecific adaptation of this simplified one-box model. (Hellweg et al. 2009; Walser et al. 2012). An example of such a steady-state model in LCA is the one-box model in USEtox for the indoor compartment that was specifically designed for gases (e.g., including degradation half-life in air) (Wenger et al. 2012). When considering the application of this model to particulates, some particle-specific properties such as agglomeration and 
sedimentation may need to be added and require further attention. Under average conditions of NP production, ventilation in manufacturing sites usually dominates other removal rates such as sedimentation or agglomeration by several orders-of-magnitude. For situations where the practitioner is not sure how to best model the process, the following considerations should be made:

- Agglomeration, which increases NP size and settling velocity, might become important in accidental situations where number concentrations greater than $10^{6} \mathrm{~cm}^{-3}$ can be reached (Hinds 1999; Walser et al. 2012). Maynard and Zimmer (Maynard 2003) proposed a numerical, timedependent model to account for the transport and fate of aerosols with regard to generation, coagulation (with subsequent agglomeration), and sedimentation through settling and diffusion. However, in well-controlled manufacturing environments, such high concentrations rarely occur (Fig. 2) and $10^{6} \mathrm{~cm}^{-3}$ is usually used as a cut-off for homogeneous agglomeration, as demonstrated by the simulations reported by Schneider et al. (2011). If agglomeration needs to be considered, the steady-state airborne concentration of NPs $\left(\mathrm{C}_{\mathrm{SS}}\right.$; number $\left.\mathrm{m}^{-3}\right)$ for a continuous emission source can be approximated in CF calculations as

$C_{\mathrm{SS}}=\frac{E}{V\left(k_{\mathrm{ex}}+2 K\right)}$

where $E$ is the emission rate (number s ${ }^{-1}$ ), $V$ is the volume of the exposure area $\left(\mathrm{m}^{3}\right), k_{\mathrm{ex}}$ is the air exchange rate of the volume in the exposure area $\left(\mathrm{s}^{-1}\right)$, and $K$ is the coagulation constant $\left(\mathrm{s}^{-1}\right)$. The coagulation coefficient is a function of particle size and can be calculated following the methods of Maynard and Zimmer (Maynard 2003) and assuming an average particle size and mass. Although the loss term due to agglomeration is a function of time and the size of the particle $\left(-\mathrm{KC}^{2}(\mathrm{t})\right)$, the term $2 K$ in a one-box model is virtually zero in steady-state mode and does not affect the steadystate concentration as long as $k_{\mathrm{ex}}$ is significantly higher.

- Gravitational settling and subsequent surface attachment are of minor importance in occupational settings because NPs typically settle at rates less than $0.1 \mathrm{~cm} \mathrm{~s}^{-1}$. However, it can be included in a one-box indoor model using Stokes' law with slip correction based on a shape factor. Shape (e.g., spherical, irregular, regular) is important for small particles when determining settling velocity and is incorporated into Stokes' law using a shape factor. The terminal settling velocity then becomes $v_{T S}=\frac{\rho_{\mathrm{p}} d_{\mathrm{e}}^{2} g C_{\mathrm{s}}}{18 \eta \eta}$, where $\rho_{\mathrm{p}}$ is the particle density, $d_{\mathrm{e}}^{2}$ the equivalent volume diameter, $g$ the gravitational force, $C_{\mathrm{s}}$ the Cunningham slip correction factor, $\eta$ the viscosity, and $\chi$ the dynamic shape factor (see Table A1). In our model, density is either calculated for spheres or adjusted for tubes according to the aspect ratio between the diameter and length of the tube via the shape factor $\chi$. The mobility diameter $d_{\mathrm{m}}$ (relevant for settling by diffusion) is preferred over the aerodynamic diameter $d_{\mathrm{a}}$ in the calculations presented in Table A2. If the mobility diameter is not directly available from the measurement campaign, the nanospecific indoor air model has been adapted to automatically calculate it as $d_{\mathrm{m}}=d_{\mathrm{a}} \cdot \sqrt{\chi \frac{C_{\mathrm{s}}\left(d_{\mathrm{a}}\right) \rho_{\mathrm{o}}}{C_{\mathrm{s}}\left(d_{\mathrm{m}}\right) \rho_{\mathrm{p}}}}$, where $\rho_{\mathrm{o}}$ is the reference density $\left(\mathrm{g} / \mathrm{cm}^{3}\right.$, Table A3 shows the interface). The losses due to gravitational settling can be included in steady-state airborne calculation as follows:

$C_{\mathrm{SS}}=\frac{E}{V\left(k_{\mathrm{ex}}+\frac{v_{T S}}{h}\right)}$

where $h(\mathrm{~m})$ is the distance between the emission source and the ground.

- Losses by molecular diffusion or thermo- or electrophoresis can occur based on Brownian motion. However, they will only be relevant for very small airborne particles $(<30 \mathrm{~nm})$. For realistic scenarios, where primary NPs either will agglomerate or be scavenged by other large particles, the loss in terms of mass will be negligible.

- Inclusion of the fractal dimension can affect the calculated mass concentration by approximately $\pm 10 \%$ for a fractal dimension of 1.5 , with much larger effects possible when the fractal dimension is increased further. However, the slight improvement to concentration estimations achieved by calculating average fractal dimensions for a mix of NPs using cluster densities does not merit the required effort within the context of life-cycle impact modeling. Moreover, the fractal dimension 
Fig. 3 An example demonstrating the calculation of relevant USEtox parameters for a hypothetical NP-X.

Conversion between concentration units and the calculation of a settling velocity for were accomplished using the unit conversion tool located in the Annex
Substance ID:
NP-X
Substance Description: $\quad$ Spherical NP
Physical Properties:
Manufacturing Specifications:

$\begin{array}{rcl}\text { Shape } & \text { spherical } \\ \text { particle diameter, } d_{\mathrm{p}} & 50 & \mathrm{~nm} \\ \rho_{\mathrm{p}} \text { (NP density) } & 5 & \mathrm{~g} . \mathrm{cm}^{-3} \\ \text { Shape factor, } \chi & 1 & \\ \text { Agglomeration Constant, } \mathrm{K} & 1.0 \mathrm{E}-16 & \mathrm{~s}^{-1} \\ \text { Mean free path, } \lambda & 100 & \mathrm{~nm}\end{array}$
Method Flame spray pyrolysis
Production rate $500 \quad$ g.h h $^{-1}$
Emission Rate, E $5 \quad$ g.h $\mathrm{h}^{-1}$
Room Volume, $V \quad 1000 \quad \mathrm{~m}^{3}$
Settling height, $h \quad 2 \quad \mathrm{~m}$
Ventilation Rate, $Q \quad 1.1 \quad \mathrm{~m}^{3} \cdot \mathrm{s}^{-1}$

Step 1: Calculate the Emission Factor (EmF)

$$
E m F=\frac{\text { emission rate }\left(\frac{\text { mass }}{\text { time }}\right)}{\text { production rate }\left(\frac{\text { mass }}{\text { time }}\right)}=\frac{0.005 \frac{\mathrm{kg}}{\mathrm{hr}}}{0.5 \frac{\mathrm{kg}}{\mathrm{hr}} \times 1 \frac{\mathrm{FU}}{\mathrm{kg}}}=0.01 \frac{\mathrm{kg}}{\mathrm{FU}}
$$

Step 2: Calculate the Steady-state Concentration $\left(C_{s s}\right)$ considering nano effects

$$
\begin{gathered}
C_{S S}=\frac{E}{V\left[k_{\text {ex }}+2 K+\frac{v_{T S}}{h}\right]}=\frac{4 \times 10^{12} \frac{\text { particles }}{\mathrm{s}}}{1000 \mathrm{~m}^{3}\left[1.1 \times 10^{-3} \mathrm{~s}^{-1}+2\left(1 \times 10^{-16} \mathrm{~s}^{-1}\right)+\frac{1.4 \times 10^{-5} \frac{\mathrm{m}}{\mathrm{s}}}{2 \mathrm{~m}}\right]} \\
=3.8 \times 10^{12} \frac{\text { particles }}{\mathrm{m}^{3}}
\end{gathered}
$$

Calculate the Fate Factor (FF)

$$
F F=\frac{C_{S S} V}{E}=\frac{1000 \mathrm{~m}^{3}\left(3.8 \times 10^{12} \frac{\text { particles }}{\mathrm{m} 3}\right)}{4 \times 10^{12} \frac{\text { particles }}{\mathrm{s}}}=9 \times 10^{2} \mathrm{~s}
$$

undergoes constant change during ambient transformations and clustering of NPs, which makes it impractical to determine in real world settings.

With the above-mentioned NP-specific extensions, the one-box model can be used to estimate indoor concentrations of NPs, provided the emission rate is known or can be calculated. The more information on particle characteristics, the more accurate the exposure estimation becomes. Without having an $\mathrm{EmF}$ available (step 1), a representative indoor concentration is required for the further calculation of nanospecific indoor exposure. Therefore, a measured or modeled (e.g., with the USEtox indoor model) steady-state concentration can then be used to calculate the intake fraction, provided the information on human uptake is available. An example calculation of $\mathrm{C}_{\mathrm{SS}}$ for a hypothetical NP is given in Fig. 3. Although we are primarily interested in the indoor emissions and concentration of synthetic NPs during their production and application and the resulting effects during worker exposure, the approach developed here can easily be adapted for consumer exposure scenarios.
Estimating worker population

Human intake via inhalation is largely a function of the indoor activities and the nanoparticle characteristics and concentration (see step 2 in Fig. 1), which will vary based on the job task and the type and effectiveness of engineering controls implemented as part of a facility's environmental health and safety (EH\&S) plan. Case-specific intake fractions can be derived directly by using workplace/breathing zone concentrations multiplied by the exposure duration, e.g., the time to produce or handle a specific amount of nanomaterial, and should be corrected for the use of any personal protective equipment (PPE) to avoid overestimation of the intake level. For the estimation of average exposure in nanoparticle production and handling, the average number of workers exposed to NPs, including duration and frequency, is relevant. However, as important as this information is, a robust set of the required data is largely unavailable to the nanoEHS research community because current regulatory practices in most countries do not require 
Table 1 Studies on the number of individuals exposed to nanomaterials in occupational settings

\begin{tabular}{|c|c|c|c|c|c|}
\hline Study focus & Year & Location & Type & $\begin{array}{l}\text { Exposed } \\
\text { workers per } \\
\text { company }\end{array}$ & Reference \\
\hline $\begin{array}{l}\text { Survey of indirectly and directly exposed } \\
\text { workers in NP manufacturing and handling } \\
\text { companies with analysis of health effects }\end{array}$ & 2013 & Taiwan & Primary & $\begin{array}{l}2,7,10,50,2 \\
\quad 3,6,2,4 \\
12,17,2,24\end{array}$ & Liao et al. (2013) \\
\hline $\begin{array}{l}\text { Worker exposure during downstream processes } \\
\text { for inclusion of nanomaterials in products by } \\
\text { industrial sector (estimated workers per } \\
\text { company) }\end{array}$ & 2013 & Netherlands & $\begin{array}{l}\text { Primary } \\
\text { (survey) }\end{array}$ & 7 & Bekker et al. (2013) \\
\hline $\begin{array}{l}\text { Total US workforce for nanotechnology based } \\
\text { on secondary data sources (does not } \\
\text { distinguish directly exposed workers; } 10 \% \text { of } \\
\text { total employment shown) }\end{array}$ & 2013 & $\begin{array}{l}\text { United } \\
\text { States }\end{array}$ & $\begin{array}{l}\text { Secondary } \\
\text { (lit. } \\
\text { review) }\end{array}$ & 25 & Frederick (2013) \\
\hline $\begin{array}{l}\text { Company-specific study of potentially exposed } \\
\text { workers at individual sites }\end{array}$ & 2011 & $\begin{array}{l}\text { United } \\
\text { States }\end{array}$ & Primary & 3 & David et al. (2011) \\
\hline Study of carbon-based nanomanufacturers & 2011 & $\begin{array}{l}\text { United } \\
\text { States }\end{array}$ & Primary & 10 & $\begin{array}{l}\text { Schubauer-Berigan et al. } \\
\text { (2011) }\end{array}$ \\
\hline $\begin{array}{l}\text { Total California (USA) workforce for } \\
\text { nanotechnology based on secondary data } \\
\text { sources (does not distinguish directly exposed } \\
\text { workers) }\end{array}$ & 2010 & $\begin{array}{l}\text { United } \\
\text { States }\end{array}$ & Secondary & 27 & $\begin{array}{l}\text { California Council on } \\
\text { Science and Technology } \\
(2010)\end{array}$ \\
\hline $\begin{array}{l}\text { Nanomanufacturing in France by industrial } \\
\text { sector (Includes both particle manufacturing } \\
\text { and product manufacturing) }\end{array}$ & 2010 & France & Primary & 38 & Honnert and Grzebyk (2013) \\
\hline Use of nanotechnology in Swiss industry & 2010 & Switzerland & Primary & 6 & Schmid et al. (2010) \\
\hline $\begin{array}{l}\text { Average Colorado (USA) workforce size for } \\
\text { nanotechnology firms (does not distinguish } \\
\text { directly exposed workers) }\end{array}$ & 2008 & $\begin{array}{l}\text { United } \\
\text { States }\end{array}$ & Secondary & 15 & $\begin{array}{l}\text { Colorado Nanotechnology } \\
\text { Alliance (2013) }\end{array}$ \\
\hline Use of nanotechnology in German industry & 2008 & Germany & Primary & 35 & $\begin{array}{l}\text { German Federal Institute for } \\
\text { Occupational Safety and } \\
\text { Health (BAuA) (2008) }\end{array}$ \\
\hline \multirow{2}{*}{$\begin{array}{l}\text { Current practices in the nanotechnology } \\
\text { workplace }\end{array}$} & 2006 & Global & Primary & 11 & Gerritzen et al. (2006) \\
\hline & & & Average & $14( \pm 13)$ & \\
\hline
\end{tabular}

companies to report such data. Any information that is available has been primarily based on voluntary surveys or secondary (literature) sources as summarized in Table 1. The level of the details provided in the studies varies from crude national estimates to company-specific information. We reviewed workplace studies for nanomaterial production and handling and found that the average number of workers to consider for potential exposure is $14 \pm 13$ workers per company (Table 1). This value provides the link between the indoor fate model and potentially exposed workforce and hence a rough starting point for impact modeling. However, it lacks several key data descriptors to accurately capture the potential impact. For instance, the average exposure time of the workforce during production and handling of NPs is not well known. A survey in 19 enterprises in WesternEurope indicated that tasks related to the use of NPs are short, e.g., Brouwer et al. (2013) (SI), reported a median duration of $11 \mathrm{~min}$ (75 percentile $35 \mathrm{~min}$ ) for such activities. Another difficulty is that chemical uptake and effects can vary within a population based on physical traits such as age, gender, and size. Thus, refined workforce data are needed at the sub-population level. Chemical uptake (and subsequently effects) will vary based on the type of NP and will require 


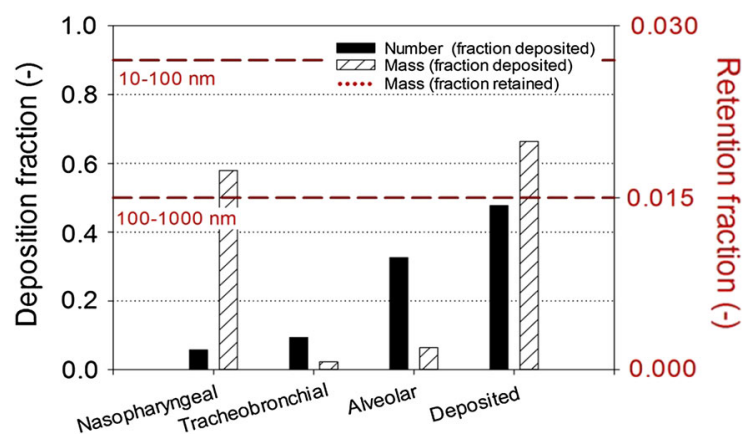

Fig. 4 Size-specific deposition of process-generated NPs (median diameter $30-70 \mathrm{~nm}$ ) in the respiratory tract (data calculated from (Elihn et al. 2011), survey of 7 Swedish Industrial Plants, $y$-axis on right. A minor part of the deposited particles remain in the body: Alveolar retention fractions are shown for two size classes of particles (10-100 nm, 100-1000 nm) calculated with MPPD (Multiple-Path Particle Dosimetry) model (v2.1), $y$-axis on left

workforce data based on the type of material being processed. This may not be simple for workers involved in the production and handling of multiple NPs throughout a typical work experience because data will be needed to understand how NPs behave during competitive uptake. Currently, data have only been reported considering production of a single NP (Bartley and Vincent 2011; Bekker et al. 2013; Brouwer et al. 2013; German Federal Institute for Occupational Safety and Health (BAuA) 2008; Honnert and Grzebyk 2013; Schmid et al. 2010; Schubauer-Berigan et al. 2011).

STEP 3-4: Intake and uptake of inhaled nanoparticles

Intake fractions for inhalation exposures in LCA are defined as the ratio of the mass of material inhaled to the mass of material released by a process or activity (e.g., $\mathrm{kg}_{\text {intake }} / \mathrm{kg}_{\text {emitted }}$ ) and are used to quantify the pollutant source-to-intake relationship (Fig. 1). Intake fractions are calculated by multiplying the predicted air concentration (FF) of the (usually gaseous) compound (corrected for use of PPE) with the number of people exposed and their daily respiratory volume. Subsequently, this number is divided by the total amount of toxic substances emitted. Traditionally, the resulting exposure factor describing intake is independent of lung physiology. This is not appropriate for small particles. In the following paragraph, we provide the concept for the calculation of the effective dose of nanoparticles in the human body upon inhalation. Given the myriad of inhaled nanoparticle forms show different biokinetics, there is not enough space in this paper to illustrate with a case study how an LCIA could take the physico-chemical characteristics of specific nanoparticles into account. In general, particle retention depends on the location of the deposition in the lung and the interaction of particles with the lung, which all vary with size, shape, elemental composition, dissolution, and surface reactivity (Braakhuis et al. 2014). Therefore, exposure factors in LCA need to be modified with nanospecific uptake fractions when assessing NPs. Deposition in the lung has been extensively studied and modeled (International Commission on Radiological Protection (ICRP) 1994). In addition to size, deposition fractions vary considerably for different types of breathing and body constitution (see Table A4, (Bartley and Vincent 2011)). The Multiple-Path Particle Dosimetry (MPPD) v2.1 model calculates a lower deposited fraction for NPs, with an estimated value of 5-10\% (Anjilvel and Asgharian 1995) than the ICRP model, which computes a value of approximately $20 \%$ (International Commission on Radiological Protection (ICRP) 1994). Key model inputs are diameter, size distribution, and density. Shape is an important, but neglected parameter in the models. Sixty percent of the deposited NPs $<100 \mathrm{~nm}$ are found in the alveolar region, with approximately $20 \%$ in the tracheobronchial part and $20 \%$ in the nasopharyngeal region of the lung (US Environmental Protection Agency 2009). Nanofibers have a different deposition pattern in the lung; Mercer et al. are reporting that $18,81.6$, and $0.6 \%$ of the nanofibers are found in the airway, the alveolar, and the subpleural regions, respectively (Mercer et al. 2008). All of these percentage values are valid for mass concentration over the specified particle size ranges and will change if different concentration units or sizes are used as shown in Fig. 4. In Fig. 4, we provide measured deposition fractions for nasopharyngeal, tracheobronchial, and the alveolar region (recalculated with MPPD from (Elihn et al. 2011)), which are shown relative to the total deposited fraction. They are rough estimates but can serve as estimates for LCA practitioners if no further information is available. Once in the lung, NPs can remain in the respiratory tract/lungs, be dissolved and absorbed, be transported with fluids, or be taken up by cells, etc. (Solomon et al. 2012). Therefore, in addition to the deposited fraction, the 
retained fraction and inclusion of shape and dissolution is needed in order to identify the relevant biological pathways and potential effects. Although retention is much lower than the deposition (Fig. 4), adverse effects can occur before particles disappear. This means the effects need to be evaluated with consideration of the contact time and location of the deposited particles. Effects can either be localized to the respiratory tract/lungs or cause systemic effects when NPs enter the blood stream and distribute throughout the body. For the localized case, shapeand size-specific deposition and retention (taking into account removal of NPs from the lungs by the mucus stream generated by the ciliary epithelium) are of importance. For systemic effects, the rate of absorption into the bloodstream is key, keeping in mind that typical translocation into the bloodstream is below $5 \%$ of the inhaled dose (Braakhuis et al. 2014). In contrast to macroparticles, NPs can be translocated into the circulatory system and accumulate in the secondary organs and tissues of the body (Kreyling et al. 2012). Surface properties (charge, morphology), elemental composition, and size influence the interaction of NPs with bodily entities, including proteins, cells, tissues, and organs. Understanding these interactions are important because human health modeling in LCA can include both local (e.g., pulmonary) and systemic (e.g., liver) effects. So, there is a need to account for these various endpoints when assessing worker exposure. This is best accomplished using a destination-based dose component for effect factors where the effective dose for an effect can is calculated as.

$D_{\mathrm{e}}=\frac{C_{\mathrm{i}} \times I H \times \boldsymbol{R} \times t}{\boldsymbol{B}}$

where $D_{\mathrm{e}}$ is the effective NP dose in the target organ for local effects or in the entire human body for systemic effects (unit must be dose), $C_{\mathrm{i}}$ is the average indoor concentration of NPs $\left(\mathrm{kg} / \mathrm{m}^{3}\right)^{1}, I H$ is the inhalation rate $\left(\mathrm{m}^{3} / \mathrm{h}\right)$, and $R$ is the total lung retention fraction (-), $t$ is the exposure time (h), and $B$ is the mass of the target organ in which toxic effects are observed (e.g., lung, entire body, etc.). The more comprehensive the characterization of the NP is in terms of agglomeration, aggregation, size, shape, and chemistry, the more robust $R$ becomes. Particle chemistry must include information on surface properties (e.g., lipophilicity), which influence phagocytic and non-phagocytic pathways of endocytosis. Unlike conventional human health impact modeling in LCA, $B$ does not have to be the body weight. However, further information is required if a dose in a target organ (other than the lung) is required. In such a case, the nanospecific lung retention factor $R$ needs to be extended with data on biokinetics that is slowly becoming available for specific nanoparticles (e.g., (Bachler et al. 2013)).

STEP 5: Nanospecific effect factors for human toxicity

Once intake occurs and the effective dose is calculated, the potential chronic and/or carcinogenic effects of a chemical can be evaluated. In LCA, exposure is averaged over the total population that is present in a certain environmental compartment and then multiplied by a unit risk per $\mathrm{kg}$ of exposure (the human EF) to calculate either a number of diseased persons or a number of years of life (quality) lost e.g., (Bare 2002; Goedkoop et al. 2009; Jolliet et al. 2003; Rosenbaum et al. 2008). It should be noted that conversion and correction factors are chosen to support best estimates in LCA as opposed to risk assessment practices of identifying the most health protective values ("precautionary principle"). Some models in LCA use DALYs to derive the unit risk to enable comparison of health effects with other impact categories (e.g., global warming). Ideally, DALYs are based on epidemiological data for the adverse effects caused by the substance under investigation. Unfortunately, such data are not available for many of the desired effects, introducing considerable uncertainty through the extrapolation of effects from in vivo animal tests or even in vitro tests to humans. A number of authors have proposed default DALYs, e.g., the proposal of Pennington et al. (Pennington et al. 2002) that differentiates between (i) irreversible/life-shortening effects, (ii) probably irreversible life-shortening effects, and (iii) reversible/non-life-shortening effects, with proposed DALYs (years per incidence) of 6.7, 0.67 , and 0.067 . Hopefully, in vitro tests in the future will support direct estimation of long-term human toxicity for nanomaterials, which would reduce the need for animal testing and circumvent the long wait time for epidemiologic data to become available. A calculation strategy for effect factors, which are referring to internal dose, is presented in Fig. 5. 
Furthermore, Quantitative Structure-Activity Relationships (QSARs), relying on a small but robust dataset, will support toxicity assessment and further reduce the efforts in laboratories (Marvin et al. 2013). However, QSARs currently cannot support derivation of human health EFs for NPs because datasets for chronic toxicity in vivo are still needed to validate the predictive models.

As previously described, mass may not be the most relevant dose descriptor for the toxicity of NPs (Donaldson and Poland 2013). The implications are that for a traditionally mass-based inventory of human health effect factors, the mass of each differently sized NP may need to be translated into more meaningful units such as surface area concentration. Moreover, grouping nanomaterials with similar modes of toxic action and/or similar toxicity profiles is commonly seen as the ideal way forward to better assess synthetic NPs (Oomen et al. 2014) because it would reduce the immense efforts of the current case by case assessments. Not only would this increase the probability of creating consistent toxicity categories, but also would facilitate ranking or scaling the NPs, which is particularly suitable for the comparative nature of LCA. For example, the availability of a high quality chronic inhalation study, together with in vitro tests for the relevant endpoint of the same nanomaterial might allow the relative comparison of other NPs with similar modes of toxic action. This could be done by first comparing the in vitro results for the two types of NPs and then correlating back to the single chronic inhalation study to estimate the unknown inhalation toxicity for the NP of interest without the need for further chronic inhalation studies. Unfortunately, nanomaterial toxicity testing has not evolved yet to the point that in vitro studies can be used to reliably predict in vivo effects (Johnston et al. 2013; Marvin et al. 2013), nor has a single material property, be it surface area, volume, or reactive oxygen species generative capacity, been shown to be perfectly correlate with observed toxic effects (Oomen et al. 2014).

A rather practical issue for LCA is the need for clear guidelines on the identity and subsequent estimation of effect factors, because the practitioner often has no access to characterization data for NPs along the life cycle. We present such guidance in a flow chart in Fig. 5. First, a clear chemical identity (Nano-CID) has to be assigned to each NP in order to make clear whether the NP is a new substance or just another form of a previously assessed substance. To illustrate, consider a NP with a core of an inherently toxic element (e.g., cadmium). It most likely exhibits a different toxicity in comparison to a particle with the same toxic element applied as a coating. Do these two NPs with the same elemental composition require a different calculation strategy for the effect factor? The differing toxicities suggest "yes," while the identical elemental compositions suggest not. The use of a Nano-CID will enable grouping of the myriad of forms of nano-sized substances, and therefore, support the calculation of grouped effect factors for NPs with similar effects. A consensus toxicity hierarchy has yet to be defined, but an example of how a classification scheme based on Nano-CID could help practitioners address typical situations while modeling NP product systems is.

1. The NP is elementally and structurally comparable to an existing Nano-CID entry: Assign the existing CID and endpoint-based EFs to the NP (Fig. 5).

2. The NP is not elementally or structurally comparable to an existing Nano-CID entry: Assign a new CID and calculate all pathway-based EFs based on best available information for the most toxic nanomaterial present (Fig. 5).

3. The NP contains a stable surface coating that is comparable to an existing Nano-CID entry: If the coating is the most toxic material present, assign the existing CID and EF to the NP; if the coating is not the most toxic material present, assign a new CID and calculate the EF based on best available information for the most toxic nanomaterial present (Fig. 5).

Two promising strategies for the estimation of effect factors are (1) collect a large amount of results from multiple studies and use statistical evaluation or (2) select a principal study for deriving a toxicological benchmark. The latter is the more common practice for regulatory purposes (Pennington et al. 2002), and therefore, from a practical point of view, it should be suitable for LCA purposes. If there is not already an effect factor available for the determined class where the investigated NP belongs to, we suggest a tiered approach like described in Fig. 5. A chronic inhalation study is the preferable starting point to derive the effect factor for human toxicity. Ideally, relevant 


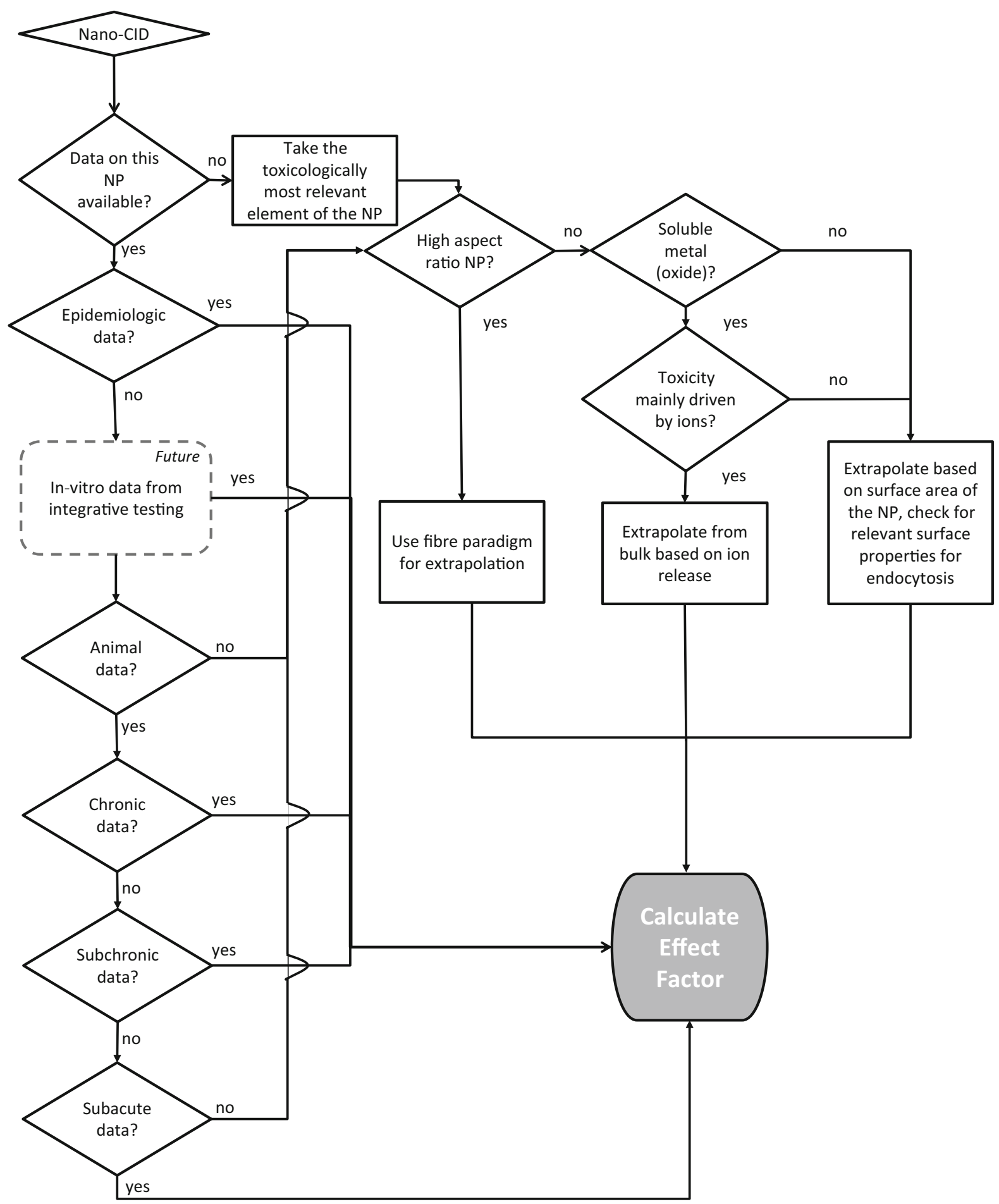

Fig. 5 Derivation strategy for the Human Health toxicity Factor ( $H E F$ human toxicity effect factor). Extrapolation is preferably based on the surface area of the NPs 
endpoints from in vitro studies will support estimation of human toxicity without animal data. If data on the particular NP are unavailable, a first approximation should consider data from similar NPs using a grouping system based on uptake and biokinetic characteristics, which are primarily defined by size, shape, and solubility of the NP. The classification into the three groups, (1) Poorly soluble, low-toxicity (PSLT) NPs, (2) Persistent high aspect ratio nanofibers, and (3) Soluble metals and metal oxides (M/MOs), is brought forward by many researchers and governmental authorities. Additional specifics have to be taken into account when working with these classes. For example, the total surface area of PSLT NPs retained in the lungs, based on the specific surface area per mass of the airborne (agglomerated) particles before inhalation, is a good predictor of the degree of toxicity (National Institute for Occupational Safety and Health NIOSH 2011). For nanofibers, asbestoslike mechanism driven by properties like length, diameter, and biopersistence (the "fibre paradigm") can be indicative of the toxic effects (Donaldson et al. 2010; Sargent et al. 2014). And finally for the M/MOs, due to their high surface-to-volume ratio, the NPs of M/MOs may release more ions than their bulk counterparts and thus be more toxic (Cho et al. 2012). How elemental properties such as oxidation state, potential for cellular uptake, etc., or the variety of different structures within these three classes play into their long-term toxicity are key information which is needed for a further refinement of Fig. 5 and a first proposal of a set of EFs for certain nanomaterials.

STEP 6: Calculation of characterization factors for indoor exposure to nanoparticles

The ultimate goal of the framework is the development of nanospecific CFs that can be used to quantify the human health impacts from an emitted quantity of NPs and therefore to support applying LCAs to nanoenabled products. The CFs may lead to impact scores for the conventional health risks such as cancer, noncancer, respiratory effects, etc. The intent is to combine all relevant nanospecific endpoints into a single CF. We have made progress toward consistent, nanospecific calculation procedures for $\mathrm{CFs}$ in this paper with the implemented unit conversion tool and evaluation of worker exposure statistics. However, we are not yet at the point where we can calculate a scientifically meaningful EFs for NPs because key information on toxicity pathways of inhaled NPs is missing, which prevents calculation of the CF. As noted above, a demonstration of the full framework with calculation of nanospecific CFs will be presented by the authors in a second publication. For this framework to be widely applicable to nano-enabled products, less time and resource intensive methods to fill toxicity data gaps will be needed. High-throughput screening methods and/or QSAR-based methods might enable grouping nanomaterials with similar hazard profiles to further simplify calculation of effect factors. However, great care is required when developing a scientifically justifiable hierarchy for grouping because all the possible combinations of physical and chemical parameters (e.g., elemental composition, different coating, variation of test media, etc.) that influence the NP toxicity can only be put into distinctive categories if a consensus can be reached between risk assessors (striving for case by case assessments) and LCA scientists (looking for generalized CFs). As another approach, it may be possible to extrapolate CFs for NPs from similar (bulk) metal particles, e.g., from epidemiologic studies of exposed people in the metal industry or close to railroads.

\section{STEP 7: Uncertainty assessment}

The final step of the framework provides a measure of the uncertainty associated with a calculated CF so it can be included in uncertainty assessments for impact assessment results. Practitioners will then understand what types of decisions for worker exposure can be supported with a NP-specific CF and what improvements are needed for the underlying data to reduce the uncertainty. This will allow existing toxicity data, as uncertain as it may be, to be used in a meaningful and transparent manner. The proposed framework for integration of NPs into the LCA framework might lead to additional uncertainties but likely does not compromise the LCIA results in view of the overall uncertainties typically associated with inventory data. The data available for derivation of CFs are not currently suitable for quantifying uncertainty assessments. Until this changes, it may be worth examining if the typical approach for estimating inventory uncertainty based on a pedigree matrix of userspecified data quality indicators can be extended to the current framework. Once data availability supports 
a quantitative uncertainty assessment of the CFs, the confidence intervals can be calculated with Monte Carlo simulations, taking into account the uncertainty distributions of each stage in the calculation of nanospecific impacts as outlined in Fig. 1.

\section{Key areas for future improvements}

An LCA on nano-enabled products should include the potential workplace health effects of inhaled nanomaterials along the supply chain of the investigated product. The first challenge to this goal is the establishment of suitable NP emission factors that can be used in LCI databases to calculate emissions based on mass production rates. These estimates of NP emissions during synthesis provide the starting point for impact assessment. The lack of unified reporting protocols for NP data in general can introduce high levels of uncertainty because emission factors can vary by orders-of-magnitude as the metrics (mass-, surface-, number concentration, etc.) are varied. This level of uncertainty must be acceptable until further information becomes available and "standard units" are introduced. A snapshot on the variety of potential exposure scenarios has been provided in the literature review in Table A5a-m. Future improvement of this work will require better characterization of the emission sources (quantity and emission factor of produced NP), a higher number of reported exposure data, a clear distinction between the background and the actual concentration of synthetic NPs, measurement campaigns over longer time periods (i.e., $>1 \mathrm{~h}$ ) with a detailed description of indoor activities, a solid description of the indoor environments, and further estimates on the number of exposed workers. Another important consideration for workplace health effects is the steady-state nature of LCA models that will not account for variable production rates, accidents, or equipment failures, i.e., a single pulse emission of NPs can be covered with risk assessment, but not with LCA. Ultimately, cumulative (modeled) exposure to NP is needed. The inclusion of all these items will help to calculate robust exposure scenarios which can then be linked to the effect factors. A final aspect of NP exposure to consider for human health is the inclusion of the fact that NPs can act as carriers for other hazardous compounds in the indoor air, resulting in toxic additional exposures.
Ideally, the NPs measured during occupational exposure studies should be identical to the NPs evaluated by nanotoxicologists. Although this is sometimes assumed during modeling and assessment out of necessity (European Commission 2012; Sung et al. 2008), it is not an accurate reflection of current practices because of the complexities that "aged" NPs add to toxicity tests. This approach could be more amenable to assessment needs if the use of wellcharacterized NPs similar to workplace NPs becomes more routine because correlation of toxicity data with characterization data could improve its applicability. As previously discussed, current trends in nanotoxicology research are focusing on a shift from in vivo to in vitro tests with increased predictability to eliminate the burdens associated with in vivo studies (Crist et al. 2013; Stone et al. 2014). As appealing as this sounds, the development of validated predictive toxicity models from in vitro data is challenging. Many of the current in vitro assays have been criticized for their reliability and comparability based on the unrealistic required particle concentrations, varying methods for estimating concentration-to-dose (i.e., particle uptake in cells), and particle transformations through agglomeration or dispersion (Lai 2012). Furthermore, NPs can behave differently in different assays, resulting in skewed results from factors such as media (reagent) interference, formation of toxic byproducts during dispersion, and misinterpretation of tissue distributions during electron microscopy analysis. Moreover, diffusion, agglomeration and kinetic properties are dependent on both the nanomaterial itself and the surrounding medium. Therefore, NPs can transform unevenly and create significantly varied effect concentrations. These transformations can lead to changes in the dose metric, i.e., number or surface concentrations instead of the tested mass concentration. These challenges must be resolved or accepted with an uncertainty spanning orders-of-magnitude prior to calculating nanospecific EFs from in vitro data for LCA.

To overcome these difficulties, a limited number of long-term, inhalation-based toxicity studies with animals could provide the necessary information to describe human toxicity potentials for NPs. Such in vivo inhalation studies might serve as a benchmark to put in vitro results for other NPs into a larger context. The goal would be to combine high- 
throughput assays, which could rank NPs according to specific modes of toxic action and results for longterm studies of well-characterized NPs to quantify effects for use in impact assessment. A recent review highlighted the fact that nanotoxicologists should focus more on lessons learned from conventional particle toxicology, because the mode of action of NPs is in most cases the same as for conventional bulk particles or incidentally and/or naturally occurring NPs (Donaldson and Poland 2013). Hence, epidemiologic studies that address cumulative longterm human health effects of incidentally produced NPs such as combustion generated NPs might be valuable for adding a background correction factor to EF calculations.

Finally, organic and most of the inorganic chemicals have their own identities, and therefore they can be picked from existing LCA databases and EFs can be easily associated to the substances. However, the EF for PM10 in LCA is a single factor which is used for particles of all forms with a characteristic length less than or equal to $10 \mu \mathrm{m}$. For synthetic NPs, the large variations in toxicity arise from the different properties and therefore, a single EF for all nanomaterials is not representative. Our proposed Nano-CID and the three groups are a first step toward practicable NP assessments in LCIA. The separate groups may facilitate read-across of toxicity within the groups. Consequently, the nanospecific contribution to the toxic effects will be better represented in the future because groups of similar nanomaterials will have their own EF, decoupled from the EF of their larger counterparts. It is important to keep in mind that for a robust comparison of the EF in LCA, consistency in the calculation of the EF is more important than a high precision or accuracy. With the LCA framework for human toxicity assessment of nanomaterials presented here, we provide a consensus between disciplines (material scientists, exposure scientists, toxicologists, and LCA researchers) that will ultimately support meaningful comparative LCAs between nano-enabled and conventional products and services.

Acknowledgments The research leading to these results has received funding from the European Union Seventh Framework Programme (FP7/2007-2013) under grant agreements no 263289 (LICARA) and no 227078 (Prosuite). We thank George Moore, LeAnna Seward, and Esther Zondervan-van den Beuken for their contributions. The U. S. Environmental Protection Agency through its Office of Research and
Development contributed to the research described here. It has not been subjected to full Agency review and therefore does not necessarily reflect the views of the Agency, and no official endorsement should be inferred.

\section{References}

Anjilvel S, Asgharian B (1995) A Multiple-Path Model of Particle Deposition in the rat lung. Toxicol Sci 28:41-50

Bachler G, von Goetz N, Hungerbuehler K (2013) A physiologically based pharmacokinetic model for ionic silver and silver nanoparticles. Int J Nanomed 8:3365

Bare J (2002) TRACI: the tool for the reduction and assessment of chemical and other environmental impacts. J Indust Ecol 6:49-78

Bartley D, Vincent JH (2011) Sampling conventions for estimating ultrafine and fine aerosol particle deposition in the human respiratory tract. Ann Occup Hyg 55:696-709

Bekker C, Brouwer DH, Tielemans E, Pronk A (2013) Industrial production and professional application of manufactured nanomaterials-enabled end products in dutch industries: potential for exposure. Ann Occup Hyg 57:314-327

Birch ME, Ku B-K, Evans DE, Ruda-Eberenz TA (2011) Exposure and emissions monitoring during carbon nanofiber production. Part I: elemental carbon and iron-soot aerosols. Ann Occup Hyg 55:mer73

Braakhuis HM, Park MV, Gosens I, De Jong WH, Cassee FR (2014) Physicochemical characteristics of nanomaterials that affect pulmonary inflammation. Part Fibr Toxicol $11: 18$

Brouwer D et al (2013) Workplace air measurements and likelihood of exposure to manufactured nano-objects, agglomerates, and aggregates. J Nanopart Res 15:1-14

California Council on Science and Technology (2010) Nanotechnology in California

Cherrie JW, MacCalman L, Fransman W, Tielemans E, Tischer M, Van Tongeren M (2011) Revisiting the effect of room size and general ventilation on the relationship between near- and far-field air concentrations. Ann Occup Hyg 55(9):1006-1015

Cho W-S et al (2012) Differential pro-inflammatory effects of metal oxide nanoparticles and their soluble ions in vitro and in vivo; zinc and copper nanoparticles, but not their ions, recruit eosinophils to the lungs. Nanotoxicology 6:22-35

Colorado Nanotechnology Alliance (2013) Doing business in Colorado. http://www.coloradonanotechnology.org

Crist RM et al (2013) Common pitfalls in nanotechnology: lessons learned from NCI's Nanotechnology Characterization Laboratory. Integr Biol 5:66-73

David RM, Nasterlack M, Engel S, Conner PR (2011) Developing a registry of workers involved in nanotechnology: BASF experiences. J Occup Environ Med 53:S32-S34

Donaldson K, Poland CA (2013) Nanotoxicity: challenging the myth of nano-specific toxicity. Curr Opin Biotechnol 24:724-734

Donaldson K, Murphy FA, Duffin R, Poland CA (2010) Asbestos, carbon nanotubes and the pleural mesothelium: a review of the hypothesis regarding the role of long fibre 
retention in the parietal pleura, inflammation and mesothelioma. Part Fibre Toxicol 7:5

Elihn K, Berg P, Lidén G (2011) Correlation between airborne particle concentrations in seven industrial plants and estimated respiratory tract deposition by number, mass and elemental composition. J Aerosol Sci 42:127-141

European Commission (2012) Second regulatory review on nanomaterials COM/2012/0572 final

Frederick S (2013) Who is the nanotechnology economy? Obstacles and methods of identifying and estimates of U.S. nano firms and workers. In: Sustainable Nanotechnology Organization (SNO) conference, Santa Barbara (United States), 2013. Santa Barbara, CA

Gerritzen G, Huang LC, Killpack K, Mircheva M, Conti J (2006) A review of current practices in the nanotechnology industry - Phase two report: Survey of current practices in the nanotechnology workplace. Produced for the International Council on Nanotechnology by the University of California, Santa Barbara. Available on http://cohesion. rice.edu/centersandinst/icon/emplibrary/ICONNanotech Survey_indexed_Full\%20Reduced.pdf

German Federal Institute for Occupational Safety and Health (BAuA) (2008) Exposure to nanomaterials in Germany

Godish T, Spengler JD (1996) Relationship between ventilation and indoor air quality: a review. Indoor Air 6:135-145

Goedkoop M, Heijungs R, Huijbregts M, Schryver AD, Struijs J, Zelm RV (2009) ReCiPe 2008-a life cycle impact assessment method which comprises harmonised category indicators at the midpoint and the endpoint level. Bilthoven (Netherlands)

Golsteijn L, Huizer D, Hauck M, van Zelm R, Huijbregts MAJ (2014) Including exposure variability in the life cycle impact assessment of indoor chemical emissions: the case of metal degreasing. Environ Int 71:36-45

Guinée JB, Heijungs R, Huppes G, Kleijn R, Koning A de, Oers L van, Wegener Sleeswijk A, Suh S, Udo de Haes HA, Bruijn H de, Duin R van, Huijbregts MAJ (2001) Handbook on Life Cycle Assessment: Operational Guide to the ISO Standards. Kluwer Academic Publishers

Hellweg S, Demou E, Bruzzi R, Meijer A, Rosenbaum RK, Huijbregts MAJ, McKone TE (2009) Integrating human indoor air pollutant exposure within life cycle impact assessment. Environ Sci Technol 43:1670-1679

Hinds WC (1999) Aerosol technology: properties, behavior, and measurement of airborne particles. Wiley, New York

Honnert B, Grzebyk M (2013) Manufactured nano-objects: an occupational survey in five industries in France. Ann Occup Hyg:met058

Huijbregts MAJ, Struijs J, Goedkoop M, Heijungs R, Jan Hendriks A, Van De Meent D (2005) Human population intake fractions and environmental fate factors of toxic pollutants in life cycle impact assessment. Chemosphere 61:1495-1504

Humbert $S$ et al (2011) Intake fraction for particulate matter: recommendations for life cycle impact assessment. Environ Sci Technol 45:4808-4816

International Commission on Radiological Protection (ICRP) (1994) Human respiratory tract model for radiological protection. Elsevier, Tarrytown

Johnston H et al (2013) Engineered nanomaterial risk. Lessons learnt from completed nanotoxicology studies: potential solutions to current and future challenges. Crit Rev Toxicol 43:1-20

Jolliet O, Margni M, Charles R, Humbert S, Payet J, Rebitzer G, Rosenbaum R (2003) IMPACT 2002+: a new life cycle impact assessment methodology. Int J LCA 8:324-330

Kreyling WG, Semmler-Behnke M, Takenaka S, Möller W (2012) Differences in the biokinetics of inhaled nano- versus micrometer-sized particles. Acc Chem Res 46:714-722

Lai DY (2012) Toward toxicity testing of nanomaterials in the 21st century: a paradigm for moving forward. Wiley Interdiscip Rev Nanomed Nanobiotechnol 4:1-15

Liao HY, Chung YT, Lai CH, Wang SL, Chiang HC, Li LA, Tsou TC, Li WF, Lee HL, Wu WT, Lin MH, Hsu JH, Ho JJ, Chen CJ, Shih TS, Lin CC, Liou SH (2013) Six-month follow-up study of health markers of nanomaterials among workers handling engineered nanomaterials. Nanotoxicol 8:100-110

Marvin HJP et al (2013) Exploring the development of a decision support system (DSS) to prioritize engineered nanoparticles for risk assessment. J Nanopart Res 15:1-13

Maynard AD (2003) Estimating aerosol surface area from number and mass concentration measurements. Ann Occup Hyg 47:123-144

Meesters JAJ, Koelmans AA, Quik JTK, Hendriks AJ, Van De Meent D (2014) Multimedia modeling of engineered nanoparticles with simpleBox4nano: model definition and evaluation. Environ Sci Technol 48(10):5726-5736

Mercer RR et al (2008) Alteration of deposition pattern and pulmonary response as a result of improved dispersion of aspirated single-walled carbon nanotubes in a mouse model. Am J Physiol Lung Cell Mol Physiol 294:L87-L97

National Institute for Occupational Safety and Health NIOSH (2011) NIOSH Current Intelligence Bulletin 63: occupational exposure to titanium dioxide

National Institute for Occupational Safety and Health NIOSH (2013) NIOSH Current Intelligence Bulletin 65: occupational exposure to carbon nanotubes and nanofibers

Oomen AG et al (2014) Concern-driven integrated approaches to nanomaterial testing and assessment-report of the NanoSafety Cluster Working Group 10. Nanotoxicology $8: 334-348$

Pennington D, Crettaz P, Tauxe A, Rhomberg L, Brand K, Jolliet O (2002) Assessing human health response in life cycle assessment using ED10s and DALYs: part 2-Noncancer effects. Risk Anal 22:947-963

Rejman J, Oberle V, Zuhorn I, Hoekstra D (2004) Size-dependent internalization of particles via the pathways of clathrin and caveolae-mediated endocytosis. Biochem J 377:159-169

Rosenbaum R et al (2008) USEtox - the UNEP-SETAC toxicity model: recommended characterisation factors for human toxicity and freshwater ecotoxicity in life cycle impact assessment. Int J LCA 13:532-546

Rosenbaum RK, Margni M, Jolliet O (2007) A flexible matrix algebra framework for the multimedia multipathway modeling of emission to impacts. Environ Int 33:624-634

Sargent LM et al (2014) Promotion of lung adenocarcinoma following inhalation exposure to multi-walled carbon nanotubes. Part Fibre Toxicol 11:3

Schmid K, Danuser B, Riediker M (2010) Nanoparticle usage and protection measures in the Manufacturing Industry-A Representative Survey. J Occup Environ Hyg 7:224-232 
Schneider T et al (2011) Conceptual model for assessment of inhalation exposure to manufactured nanoparticles. J Expo Sci Environ Epidemiol 21:450-463

Schubauer-Berigan MK, Dahm MM, Yencken MS (2011) Engineered carbonaceous nanomaterials manufacturers in the United States: workforce size, characteristics, and feasibility of epidemiologic studies. J Occup Environ Med 53:S62-S67

Scientific Committee on Emerging and Newly Identified Health Risks (SCENIHR) (2010) Scientific basis for the definition of the term "Nanomaterial". SCENIHR, Brussels

Solomon P et al (2012) Macroscopic to microscopic scales of particle dosimetry: from source to fate in the body. Air Qual Atmos Health 5:169-187

Stone V et al (2014) ITS-NANO-Prioritising nanosafety research to develop a stakeholder driven intelligent testing strategy Part Fibr Toxicol 11:9
Sung JH et al (2008) Lung Function Changes in SpragueDawley Rats After Prolonged Inhalation Exposure to Silver Nanoparticles. Inhal Toxicol 20:567-574

US Environmental Protection Agency (2009) Integrated science assessment for particulate matter (Final Report). Washington, DC

van Zelm R et al (2008) European characterization factors for human health damage of PM10 and ozone in life cycle impact assessment. Atmos Environ 42:441-453

Walser T, Hellweg S, Juraske R, Luechinger NA, Wang J, Fierz M (2012) Exposure to engineered nanoparticles: Model and measurements for accident situations in laboratories Sci Tot Env 420

Wenger Y, Li D, Jolliet O (2012) Indoor intake fraction considering surface sorption of air organic compounds for life cycle assessment. Int J LCA 17:919-931 